Alain-Marc Rieu

Professeur, Faculté de philosophie, Université de Lyon - Jean Moulin \& Institut d'Asie Orientale (CNRS UMR 5062) ENS-Ish

\title{
Géostratégie de la recherche et de l'innovation
}

Revue Hermès, Paris, CNRS Editions, n 50,2008 , p. 67-73

\section{Une autre géopolitique}

Deux systèmes de Recherche et Développement sont aujourd'hui effectivement en mesure d'organiser et gérer, de financer et réaliser des politiques de recherche de grande échelle, ayant un impact mondial, les Etats-Unis et le Japon. C'est par rapport à ces deux systèmes que toutes les stratégies de développement scientifique et technique se positionnent aujourd'hui, que leurs concepteurs l'admettent ou non. Une évolution majeure est la tentative de concevoir et d'organiser au niveau de l'Union européenne un troisième système de recherche scientifique et technique susceptible d'avoir un impact mondial. Toutes ces politiques ont clairement des visées hégémoniques, même si les objectifs sont différents, depuis une affirmation de souveraineté afin de maîtriser les conditions du développement économique et social d'un Etat ou d'une région, jusqu'à la volonté d'établir une trajectoire techno-scientifique contraignant les autres nations à se situer en position de rattrapage par rapport à elle.

Cette étude ne porte pas sur les stratégies de rattrapage mais sur la géopolitique de I'hégémonie par et dans la recherche techno-scientifique, sur les différents agencements institutionnels à la base de cette hégémonie. Pour le moment, la Russie, la Chine et l'Inde développent des politiques de recherche qui sont à la fois des affirmations de souveraineté nationale et des stratégies de rattrapage. En 2000, I'ambition de I'Union européenne exprimée par la Stratégie de Lisbonne pour 2010 était de « devenir l'économie de la connaissance la plus compétitive et la plus dynamique du monde, capable d'une croissance économique durable accompagnée d'une amélioration quantitative et qualitative de l'emploi et d'une plus grande cohésion sociale ». Cet objectif est aujourd'hui hors de portée, sans que les causes de cet échec soient pleinement étudiées ${ }^{1}$.

Reste à savoir comment une politique européenne de la recherche pourrait se positionner par rapport aux politiques mises en œuvre au Japon et aux Etats-Unis. Une

\footnotetext{
1 Voir mon article «Reconstruire I'Europe au-delà du oui et du non », Le monde, 6 septembre 2005. Il associe la question de la recherche et la construction institutionnelle. Voir http://w3.ens-lsh.fr/amrieu/; rubrique : Recherche. On line documents. Voir aussi Emerging Knowledge Societies in the EU and Japan: reconfiguring collaboration in the Social Sciences and the Humanities. Tokyo, July 2006 (http://ipn.cec.eu.int/data/current/Rieu EmergingKnowledgeSocieties.pdf).
} 
chose est claire : ce n'est pas une simple affaire de volonté politique, de coopération, de financement. Concevoir une telle politique suppose d'abord une intelligence commune des processus concernés. Une des façons d'y parvenir est de disposer d'une compréhension adaptée des politiques de recherche américaine et japonaise qui prenne en compte leurs différences, leurs interactions et leurs confrontations. C'est nécessaire si I'on veut comprendre comment s'exprime aujourd'hui la compétition entre les pays industriels avancés, quels sont les enjeux d'une recherche de position hégémonique par la recherche et l'innovation.

\section{Un double processus mimétique}

La compétition engagée est d'autant plus forte aujourd'hui qu'elle repose sur un constat formulé au Japon au début des années $1990^{2}$ : le monde était entré dans un processus mimétique. Dans un marché potentiellement ouvert, chaque économie était désormais en mesure d'identifier et de produire les biens et les services lui permettant de concurrencer ses partenaires et ses rivaux sur leur marché intérieur et sur le marché mondial. Ce processus paraissait sans limites, capable de révolutionner l'économie mondiale, en redistribuant la richesse des uns (les pays industrialisés depuis le XIX ${ }^{\circ}$ siècle, y compris le Japon) et en induisant I'enrichissement des autres, les pays émergents. La grande redistribution commençait, bien différente du « nouvel ordre mondial » promu au même moment par les Etats-Unis à la fin de la première guerre d'Irak. Ce « nouvel ordre » relevait en effet d'une géopolitique de la puissance économique, politique et militaire, version de I'ordre mondial établi en 1945 . Vue du Japon, à la fin de la guerre froide et au moment du décollage de la Chine, la globalisation serait l'âge d'une course généralisée au rattrapage.

Face à ce diagnostic, les élites japonaises entreprirent d'extraire le plus vite possible le pays de ce processus mimétique en établissant les bases d'un autre système socio-économique pour atteindre une avance, un « avantage comparatif 3 », reposant sur la recherche et l'innovation. Une « économie de l'innovation » n'était pas une idée nouvelle : elle datait des années 1970, de l'émergence des «nouvelles technologies » et de la prise de conscience de leur impact économique et social. Mais le Japon est le premier pays à concevoir une mutation du rôle et de l'organisation de la R\&D comme une réponse à la globalisation, à entreprendre une refonte de son système de Recherche et d'Education, une réforme de son économie et de son système institutionnel, afin d'ouvrir

\footnotetext{
2 Voir « Le Japon comme société de connaissance : quelles leçons pour la France ? » dans J.F. Sabouret (sous la direction de), L'empire de l'intelligence : les politiques de recherche japonaises depuis 1945, Paris, CNRS Editions, 2007, p 185-216.

3 Michael Porter, The competitive advantage of Nations, New York, Free Press, 1990.
} 
un nouveau cycle de développement social et économique où il devancerait la globalisation mimétique pour s'affranchir de ses conséquences ${ }^{4}$.

Dans les années 1990, le Japon inaugurait en fait un deuxième processus mimétique. En effet, quinze ans plus tard, tous les pays industriels ont adopté ce diagnostic et sont engagés dans un processus mimétique à deux faces : I'une repose sur la globalisation de la production des biens et des services en direction les pays émergents mais à destination des pays industrialisés. L'autre repose sur l'idée que la Recherche et Développement permettra aux pays industrialisés de maintenir leur « avantage compétitif » grâce à la conception et à la production de biens de valeur ajoutée croissante, qui seront transférées à leur tour vers des pays supposés ainsi indéfiniment émergents, en situation de rattrapage. De ce double processus devrait résulter un « cercle vertueux », une « spirale positive », compensant chez tout le monde les effets destructeurs par la création de nouvelles activités, par « percolation » progressive.

Ce double processus mimétique est à la fois maelstrom et tsunami. Il aspire les sociétés les unes après les autres et les submerge en même temps. Il marginalise des populations dans les pays exclus du processus comme dans les pays où ce processus se produit. La radicalisation du terrorisme date de la même époque : elle est un rejet des sociétés où la recherche scientifique brise des tabous millénaires concernant I'homme, la société, le vivant, la nature ; elle est une révolte contre l'exclusion intense qu'engendre cette avancée qui renforce la position hégémonique des sociétés industrielles.

\section{Eléments d'une géopolitique de la recherche}

Une clarification est nécessaire. Ce qu'on nomme «politique de recherche » est confus, trop souvent confondu avec une politique industrielle de l'innovation, une planification des investissements dans la Recherche et Développement, une stratégie de recherche organisée par un Etat ou un gouvernement dans une logique de rattrapage. Ce qu'on observe aux Etats-Unis et au Japon est d'une tout autre ampleur. Une politique scientifique et technique suppose d'abord un agencement institutionnel d'une grande complexité où le rôle de l'Etat est profondément transformé. Elle est conçue et mise en œuvre par un ensemble d'institutions associant des savoirs et des compétences dans un grand nombre de domaines. Elle exige des ressources humaines autant que financières, des universités puissantes aussi bien qu'un tissu d'entreprises de conception, de production et de conseil, des organismes de formation, de gestion et de régulation, un cadre juridique (dont la propriété industrielle), un Etat et une administration adaptés. II

\footnotetext{
4 Voir « Japan as a techno-scientific society. The new role of R\&D » Tokyo, National Institute for Research Advancement Review, Autumn 1996 (http://www.nira.go.jp/publ/review/96autumn/rieu.html).
} 
faut y ajouter le poids d'une histoire, des pratiques et des pouvoirs établis. Tel est le dispositif institutionnel distribué, le champ communicationnel, dans lequel se construit une politique de recherche se donnant pour but de créer les bases de long terme du développement social et économique d'une région ou d'une nation. La formation d'un tel dispositif est indissociable de l'intelligence qu'on en a et des modes de communication qu'elle suppose. Cette intelligence outrepasse les démarcations établies entre les sciences de l'homme et de la société. Elle ne peut pas se réduire à la plus avancée d'entre elles, l'économie de l'innovation ${ }^{5}$. Enfin, cet agrégat n'est jamais cohérent, mais son degré de cohérence et le type de gouvernance qui en résulte, conditionnent sa capacité à concevoir et surtout à performer une politique de recherche. La gouvernance d'une politique de recherche ne peut donc pas être entre les mains d'un gouvernement et une bureaucratie d'Etat. Sa conception exige une négociation complexe entre les différents acteurs et secteurs de sa réalisation ; elle organise ainsi les réseaux qui la mettront en œuvre.

On nomme communément cet agrégat un « système de Recherche et Développement ». Il peut être national, aussi bien que régional ou local. Cette notion est désormais trop limitée. Elle est principalement concernée par la cohérence négociée entre les différents acteurs de la R\&D au niveau national ou régional. Son modèle le plus explicite est le «triple hélix ${ }^{6} », A D N$ des sociétés industrielles avancées, associant dans une dynamique commune, trois secteurs distincts, les universités, l'Etat et les entreprises. Une autre dimension doit être prise en considération : les interactions et les interférences entre les différents systèmes de R\&D. En effet, ces systèmes sont en intense concurrence les uns avec les autres. Même s'ils expriment la souveraineté de chaque Etat, ils ne sont pas isolés les uns des autres, mais au contraire imbriqués, en situation de dépendance et de coopération. Ils sont autant prisonniers de l'histoire qui les distingue que des enjeux hégémoniques qui les opposent. Toute modification ou réforme de I'un agit sur les autres, peut bloquer certains, entraver ou favoriser le développement d'un autre.

Deux mutations, à fois conceptuelles et institutionnelles, doivent être prises en compte. D'abord, le cas du Japon et (de certains états) des Etats-Unis montre qu'un système de Recherche et Développement ne vient pas s'ajouter aux systèmes politique, économique ou social existants : il en est aujourd'hui non seulement l'expression mais l'infrastructure commune. C'est pourquoi l'environnement institutionnel joue un rôle

\footnotetext{
5 Je renvoie en France aux travaux de Robert Boyer, Michel Callon et Dominique Foray.

6 L. Leydesdorff \& H. Etzkowitz, "Emergence of a triple helix of university-industry-government relations", Science and public policy, vol. 23, n 5, 1996, p 279-286.
} 
déterminant. C'est à ce niveau qu'opèrent les ouvertures et les blocages : les relations entre l'Etat, les milieux de la recherche et les entreprises, le degré d'autonomie des universités, la conception de l'« intérêt national » promue par la bureaucratie d'Etat, les valeurs établies et le système juridique, le niveau de culture scientifique et technique, sont autant de contraintes et d'opportunités. Il s'ensuit un point essentiel : la dimension du territoire, le nombre d'habitants et la démographie, importent moins que la cohérence et la gouvernance du système de R\&D que les Etats ou les régions sont capables d'organiser et mettre en œuvre. Les exemples abondent : (presque) tout oppose le Japon et les Etats-Unis, le système de R\&D de la Suisse est plus performant que le système français, etc. On parle toujours des budgets, du pourcentage du PIB consacré à la recherche, du montant par habitant. Ces données mesurent certes des conditions nécessaires, mais certainement pas suffisantes. Elles expriment d'abord que les modèles économiques sont actuellement les mieux à même de décrire et de gérer ces systèmes parce qu'ils les rendent gouvernables.

Penser la recherche du point de vue de l'environnement institutionnel suppose une deuxième mutation, plus perturbante : il faut apprendre à penser la recherche scientifique et technique en dehors des laboratoires, des universités, des revues spécialisées, en plus du travail des chercheurs, des découvertes et des innovations. Le rôle et I'organisation de la recherche ont en effet connu depuis les années 1980 une mutation tout aussi importante que les découvertes. Cette mutation du régime épistémique s'est avérée peut-être même plus décisive parce qu'elle explique l'ampleur des innovations qui transforment nos sociétés et leurs relations. C'est à cette condition qu'une géopolitique de la recherche et de l'innovation devient pertinente. L'exposer, c'est décrire des processus et des conflits de pouvoir, c'est poser le problème de l'intelligence et de la gouvernance des politiques de recherche.

\section{Interférences et conflits entre systèmes de R\&D}

Comprendre ce qu'est la géopolitique de la recherche et de l'innovation exige finalement d'expliquer pourquoi le Japon et les Etats-Unis sont aujourd'hui en situation hégémonique. Le Japon est d'abord le pays qui, en dehors de l'Occident, est parvenu au $\mathrm{XIX}^{\circ}$ siècle à assimiler les technologies modernes et à se transformer en une puissance industrielle et militaire capable au $X X^{\circ}$ siècle de contester le pouvoir des Européens et des Américains sur le continent asiatique et dans le Pacifique. La « guerre totale » dans laquelle s'était lancé I'Etat japonais dès les années 1930 avait contraint les Etats-Unis à entrer en guerre et à développer des techniques d'organisation, de communication, d'armement et de production à une échelle sans précédant. Après 1945, écrasé et humilié, le Japon a été reconstruit sous tutelle américaine selon l'opposition suivante : 
aux Etats-Unis, la science fondamentale ; au Japon, I'innovation technique et industrielle. Le Japon s'est donc reconstruit autour d'un système national d'innovation à des fins de rattrapage.

Survint dans les années 1970 un événement disruptif majeur, la première crise de l'énergie et des matières premières. Chaque pays a réagi en fonction de la trajectoire évolutive dont il est le produit7. Pour les Etats-Unis, le chantage à l'accès au pétrole et aux matières premières fut interprété comme une atteinte à leur souveraineté et à leur hégémonie, comme une tentative de contrôle du monde «libre ». Leur supériorité militaire, monétaire et industrielle, le pouvoir politique en résultant, leur ont permis de sécuriser l'accès aux ressources naturelles et de refouler le problème posé jusqu'au milieu des années 2000. Comment le Japon a-t-il réagi ? La hausse des matières premières, leur rareté potentielle furent interprétées comme une mise en cause de leur reconstruction d'après-guerre, comme une menace envers l'autonomie nationale. Le choc fut immense. Il n'était pas simplement énergétique ou économique : la menace était aussi grave que le risque de colonisation au XIXº siècle. Les élites japonaises ont réagi en transformant leur industrie, en intensifiant et en généralisant l'innovation par la conception et la production de biens incorporant la dernière vague technologique née aux Etats-Unis, l'informatique. En quelques années, les biens « de haute valeur ajoutée » des firmes japonaises concurrencèrent partout dans le monde les produits américaines et européennes.

Les Etats-Unis ont alors pris diverses mesures. Deux d'entre elles eurent un immense impact. La première est la transformation du régime de la propriété industrielle, le célèbre Bayh-Dole Act de 1980. Désormais les découvertes financées par les budgets publics pouvaient devenir la propriété des universités et des chercheurs qui les réalisaient. Des brevets pouvaient être passés et des licences exclusives accordées aux entreprises privées, en particulier les petites entreprises. Les universités pouvaient ouvrir I'accès aux connaissances qu'elles produisaient grâce au cadre légal établissant les droits respectifs des différents partenaires. Elles pouvaient ainsi augmenter leurs revenus et investir dans la recherche. L'université ne sera plus jamais la même. Les universités américaines ajoutèrent ainsi à leur puissance scientifique un pouvoir et une responsabilité économiques qui intensifièrent en quelques années aussi bien les innovations que la création d'industries. Les « nouvelles technologies » furent les principales bénéficiaires de cette mutation. A partir des années 1990, l'industrie japonaise était concurrencée de toute part. Les Etats-Unis venaient de bouleverser leur système de Recherche scientifique et de Développement techno-industriel : ils étaient

\footnotetext{
7 L'étude de la trajectoire évolutive du Japon est l'objet de mon livre Savoir et pouvoir dans la modernisation du Japon, Paris, P.U.F., 2001.
} 
passés d'une hégémonie de $1^{\circ}$ génération, d'une R\&D encadré par l'Etat, les entreprises énergétiques et la Défense, à une hégémonie de $2^{\circ}$ génération, celle du « triple hélix ${ }^{8}$.

Mais I'administration républicaine de Ronald Reagan prit une deuxième mesure fondée sur la tutelle imposée au Japon d'après-guerre : elle décida d'agir sur le taux de change du Yen par rapport au Dollar. La réévaluation du Yen eut lieu en 1985 par les Accords du Plazza Hotel à New York ; elle fut aggravée par la crise boursière de 19879 . Le coup porté à la compétitivité japonaise plongea finalement le Japon dans une crise qui allait progressivement déconstruire son système social et économique, ce dont il cherche encore à se remettre. Il faut insister sur l'extrême violence de la décision américaine et sur l'ampleur de ses conséquences au Japon et ailleurs dans le monde.

Les Japonais ne pouvaient se protéger contre une telle attaque. Ils étaient isolés en Asie par la tutelle des Etats-Unis et par la haine née des crimes de guerre commis par leurs armées pendant les années 1930 et 40. La crise dans laquelle le pays plongeait parut sans fond jusqu'au moment, à la fin des années 1990, où elle fut interprétée comme une transition vers un autre système socio-économique. Le Japon disposait en effet d'un modèle qui avait fait ses preuves à la fin des années 1970. Dès le début des années 1990, se forme au MITI le projet d'entreprendre cette fois une réforme complète du système national de Recherche, d'Education et d'Innovation afin d'établir les bases d'un autre système économique et social. Une loi-cadre votée en 1995 conduisit à la conception et à la réalisation de trois «Basic Plan for Science and Technology » successifs, d'une ambition croissante, le dernier lancé en 2006. La productivité de ces plans est inférieure aux prévisions officielles ; le proche avenir permettra d'évaluer leur impact. Mais un fait est certain : le Japon a su construire une politique scientifique et technologique qui lui est propre, en pleine collaboration avec la recherche mondiale, y compris celle des Etats-Unis dont il n'entend pas se passer. Le Japon n'a pas d'autre ambition hégémonique qu'établir les bases de long terme de ses capacités d'innovation et de développement. Sur la base de ce système, il transforme d'abord ses relations technologiques et commerciales, politiques ensuite, avec ses voisins d'Asie de l'Est. Il entend ainsi se libérer de la tutelle des Etats-Unis qui ont grand besoin de lui pour garder une présence et une capacité de contrôle en Asie de I'Est. Peut-être est-ce déjà trop tard.

Quant aux Etats-Unis, leur hégémonie scientifique, technologique et industrielle révèle à partir des années 2000 des lignes de tension et de faiblesse. Je retiens l'enjeu géopolitique le plus manifeste. La montée en puissance du secteur des « nouvelles technologies » fut aussi l'émergence d'un nouveau pouvoir financier et industriel. Il

\footnotetext{
8 La contrainte environnementale introduit désormais une troisième géopolitique.

9 En une année, le taux de change du Yen passa de 260 à 150 pour 1 Dollar. L'excédent commercial japonais passa de 912 milliards de Yen fin 1985 à moins de 500 milliards fin 1989. La hausse du Yen provoqua une surchauffe de l'économie japonaise que le Ministère des finances ne put contrôler. La bulle éclata en janvier 1990 : le Nikkei perdit $40 \%$ de sa valeur en un an. Les Etats-Unis demandait une réévaluation du Yen depuis plusieurs années.
} 
engendra une intense « clusterisation », une reconfiguration du territoire national autour des lieux où se concentraient le capital et les compétences, les meilleures universités, les entreprises et les services les plus innovants. De nouvelles valeurs apparaissaient, ainsi qu'une autre conception de la nation et des relations internationales. Ce nouveau pouvoir s'est finalement opposé au pouvoir en place depuis la deuxième guerre mondiale, associant les industries militaires, le Pentagone, l'administration centrale et les industries de l'énergie et des matières premières. Ce pouvoir exprime d'autres valeurs, d'autres intérêts, une autre conception des Etats-Unis et des relations internationales, une autre configuration économique du territoire. Depuis les grandes bases militaires jusqu'aux milieux ruraux, dans les périphéries lointaines des nouvelles technologies, une vaste coalition s'est retrouvée derrière le couple G.W. Bush-Cheney. Impossible de dire fin 2007 comment cette tension va évoluer, peut se dénouer. Mais le conflit à l'intérieur des Etats-Unis, entre ces deux géopolitiques, a un impact mondial, y compris sur les politiques de recherche.

\section{Conclusion}

On comprend ainsi comment deux systèmes de Recherche et Développement profondément différents se trouvent aujourd'hui en position hégémonique. Leurs interférences et conflits ont intensifié leur développement et progressivement rejeté le reste du monde en position de spectateurs, en situation de rattrapage et de dépendance ou d'exclusion. Une Europe de la recherche ne peut se former qu'en partant de ce constat, qu'en analysant la source, les enjeux et les conséquences de cette géopolitique de la recherche et de l'innovation. Il faut passer par là pour disposer d'une intelligence adaptée. Il faut en même temps construire une autre épistémologie. Des travaux sont engagés, des évolutions institutionnelles imaginées. Travailler avec des spécialistes japonais est une façon d'avancer. J'espère y avoir contribué. 